\title{
A Systematic Review of Studies Published between 2016 and 2019 on the Effectiveness and Efficacy of Pneumococcal Vaccination on Pneumonia and Invasive Pneumococcal Disease in an Elderly Population
}

\author{
Jacob Dag Berild ${ }^{1, *}$, Brita Askeland Winje ${ }^{1}$, Didrik Frimann Vestrheim ${ }^{1}$, \\ Hans-Christian Slotved ${ }^{2} @$, Palle Valentiner-Branth ${ }^{3}$, Adam Roth ${ }^{4}$ and Jann Storsäter ${ }^{4}$ \\ 1 Department of Vaccine Preventable Diseases, Division of Infection Control and Environmental Health, \\ Norwegian Institute of Public Health, 0213 Oslo, Norway; Brita.Askeland.Winje@fhi.no (B.A.W.); \\ DidrikFrimann.Vestrheim@fhi.no (D.F.V.) \\ 2 Department of Bacteria, Parasites and Fungi, Statens Serum Institute, 2300 Copenhagen, Denmark; \\ hcs@ssi.dk \\ 3 Infectious Disease Epidemiology \& Prevention, Statens Serum Institute, 2300 Copenhagen, Denmark; \\ pvb@ssi.dk \\ 4 Unit for Vaccination Programmes, Public Health Agency of Sweden, 17182 Solna, Sweden; \\ adam.roth@folkhalsomyndigheten.se (A.R.); jann.storsater@folkhalsomyndigheten.se (J.S.) \\ * Correspondence: jacobdag.berild@fhi.no
}

Received: 6 March 2020; Accepted: 1 April 2020; Published: 3 April 2020

check for updates

\begin{abstract}
Adult vaccination is high on the agenda in many countries. Two different vaccines are available for the prevention of pneumococcal disease in adults: a 23-valent polysaccharide vaccine (PPV23), and a 13-valent conjugated vaccine (PCV13). The objective of this review is to update the evidence base for vaccine efficacy and effectiveness of PPV23 and PCV13 against invasive pneumococcal disease and pneumonia among an unselected elderly population. We systematically searched for clinical trials and observational studies published between January 12016 and April 17 2019 in Pubmed, Embase, Cinahl, Web of Science, Epistemonikos and Cochrane databases. Risk of bias was assessed using Cochrane Risk of Bias tool for and the Newcastle-Ottawa Scale. Results were stratified by vaccine type and outcome. We identified nine studies on PCV13 and six on PPV23. No new randomized clinical trials were identified. Due to different outcomes, it was not possible to do a meta-analysis. New high-quality observational studies indicate protective vaccine effectiveness for both vaccines against vaccine type pneumonia. Our estimates for the protective vaccine efficacy and effectiveness (VE) of PPV23 on pneumonia and pneumococcal pneumonia overlap with results from previously published reviews. Some of the results indicate that the effectiveness of the PPV23 is best in younger age groups, and that it decreases over time.
\end{abstract}

Keywords: pneumococcal vaccines; review; invasive pneumococcal disease; pneumonia; elderly

\section{Introduction}

Streptococcus pneumoniae is a leading cause of mortality and morbidity in the elderly population. The most severe form of pneumococcal disease is invasive pneumococcal disease (IPD). Additionally, S. pneumoniae can cause non-invasive disease such as pneumonia, sinusitis and otitis media. Estimates show that the number of hospitalizations due to pneumococcal pneumonia will double in the US from 2004 to 2040 without any interventions, and that this will lead to a $\$ 2.5$ billion increase in health 
care expenditure [1]. In an effort to reduce this burden, two different vaccines have been developed - a 23-valent polysaccharide vaccine (PPV23, Pneumovax 23, Sanofi Pasteur, MSD) and a 13-valent conjugate vaccine (PCV13, Prevenar13, Pfizer). With the exception of serotype 6A, all serotypes in PCV13 are also included in PPV23.

The randomized placebo-controlled trial named CAPITA demonstrated the efficacy of PCV13 in adults aged $\geq 65$ years. The study was conducted in the Netherlands, included almost 85,000 participants, and found a modified intention-to-treat vaccine efficacy of 37.7\% (95\% CI: 14.3 to 55.1 ) against the first episode of vaccine-type community acquired pneumococcal pneumonia (VT-CAP) and $75.8 \%$ (95\% CI: 46.5 to 90.3) against the first episode of vaccine-type invasive pneumococcal disease (VT-IPD) [2].

In 2016 and 2017, five different systematic reviews evaluated the vaccine efficacy and effectiveness (hereafter both will be abbreviated VE) of the PPV23 vaccine [3-7] (Table S1). The pooled VE on invasive pneumococcal disease was estimated as 73\% in randomized controlled trials (RCTs) [4] and as $45-59 \%$ in observational studies $[4,5]$ (Table S1). However, the estimated collected body of evidence on pneumococcal pneumonia varied greatly between the reviews-some reviews indicated a statistically significant VE [4,5,7], while others did not [3,6] (Table S1). The disparate results reflect different inclusion and exclusion criteria for studies and designs. Many of the reviews also reported on all-cause pneumonia [3,5-7], but none of the systematic reviews reported on vaccine-type (VT) pneumonia. Against this background the Norwegian Institute of Public Health, Statens Serum Institute in Denmark and the Public Health Agency of Sweden, we conducted a systematic review to update the evidence base for $\mathrm{VE}$ of pneumococcal vaccines against invasive pneumococcal disease and pneumonia in a general elderly population.

\section{Materials and Methods}

\subsection{Literature Search}

We systematically searched for eligible publications in Pubmed, Embase, Cinahl, Web of Science, Epistemonikos and Cochrane databases.

To avoid repeating previous work [3-7], we only considered publications from January 12016 and onwards.

Eligible studies were original reports on VE of the PCV13 or PPV23 in a general elderly population. Thus, we excluded studies that only included high-risk patients. We did not choose a specific age cut-off in order to avoid missing any important publications. The comparator was no vaccine or a placebo vaccine. We only considered studies reporting pneumonia and/or IPD as clinical outcomes, including all-cause, pneumococcal and/or serotype-specific disease. We did not set an a priori case definition for pneumonia or IPD, but used the case definitions used by respective authors. Both observational studies and clinical trials were included. Publication language was restricted to English, German, French, Spanish, Dutch, Danish, Norwegian or Swedish language. Furthermore, we excluded case reports, animal, modelling, immunogenicity, and carriage studies, health economic evaluations, and studies on the indirect VE of pneumococcal vaccination. Appendix A contains the complete electronic search strategy.

We conducted the search August 232018 with the support from a scientific librarian. Two reviewers (J.D.B, J.S.) independently extracted eligible studies according to the PICO (Population, Intervention, Comparator and Outcome) (Appendix C). We first screened by title and abstract, then by reading the full text. Any disagreement was resolved in consensus, and if necessary, with the help of a third reviewer (B.A.W.). We repeated this process on April 17 2019, to check for new publications. 


\subsection{Data Extraction}

J.D.B and B.A.W. extracted data from eligible studies using a predefined form (Appendix B). The main outcomes were VE on pneumonia and/or IPD, including all-cause pneumococcal and serotype-specific disease. Results were stratified by study design, vaccine type, outcome and age.

\subsection{Quality Assessment}

We assessed the quality of randomized trials using Cochrane Collaboration's tool for assessing risk of bias [8], and observational studies using the Newcastle-Ottawa Scale (NOS) $[9,10]$.

\subsection{Analysis}

VE estimates from the studies are presented individually.

\section{Results}

After the removal of duplicates we identified 803 records, of which 771 were excluded based on title and abstract (Figure 1). After full text review, we included 12 studies (8 on PCV13 and 4 on PPV23). The updated search conducted April 172019 yielded 257 new records, from which one new PCV13 study was included. We also included two additional PPV23 studies found through other sources. Table 1 provides an overview of the included studies.

\subsection{Characteristics}

\subsubsection{Conjugate Vaccine}

Nine studies reported on PCV13 VE [11-19] (Table 1). Five of these were post-hoc analyses based on CAPITA data [11-15]. Besides the post-hoc studies, we identified two cohort studies, one from Spain [16] and one from Germany [17], and two studies using test-negative design (TND), one from the US [18] and one from Italy [19]. The Spanish cohort study used routine data collected in 2015 to identify all-cause pneumonia and pneumococcal pneumonia cases. Cohort members were observed for almost 2,000,000 person-years. However, only about 7000 of these were in PCV13-vaccinated persons [16].

The German cohort study used routine insurance data to identify 11,395 people vaccinated with PCV13, of a cohort of more than 500,000 inhabitants living in Saxony between 2014 and 2016 [17]. The American TND study included 2034 CAP hospitalizations and identified 68 VT-CAP cases using a serotype-specific urinary antigen test developed by Pfizer. The remaining non VT-CAP patients were controls [18]. The Italian TND study identified 1867 eligible CAP patients between 2013 and 2015, but due to a low participation rate, they only included 182 CAP patients in their VE analysis. Using a range of different diagnostic tools, they identified 59 pneumococcal CAP cases, of which 39 were VT-CAP [19]. 


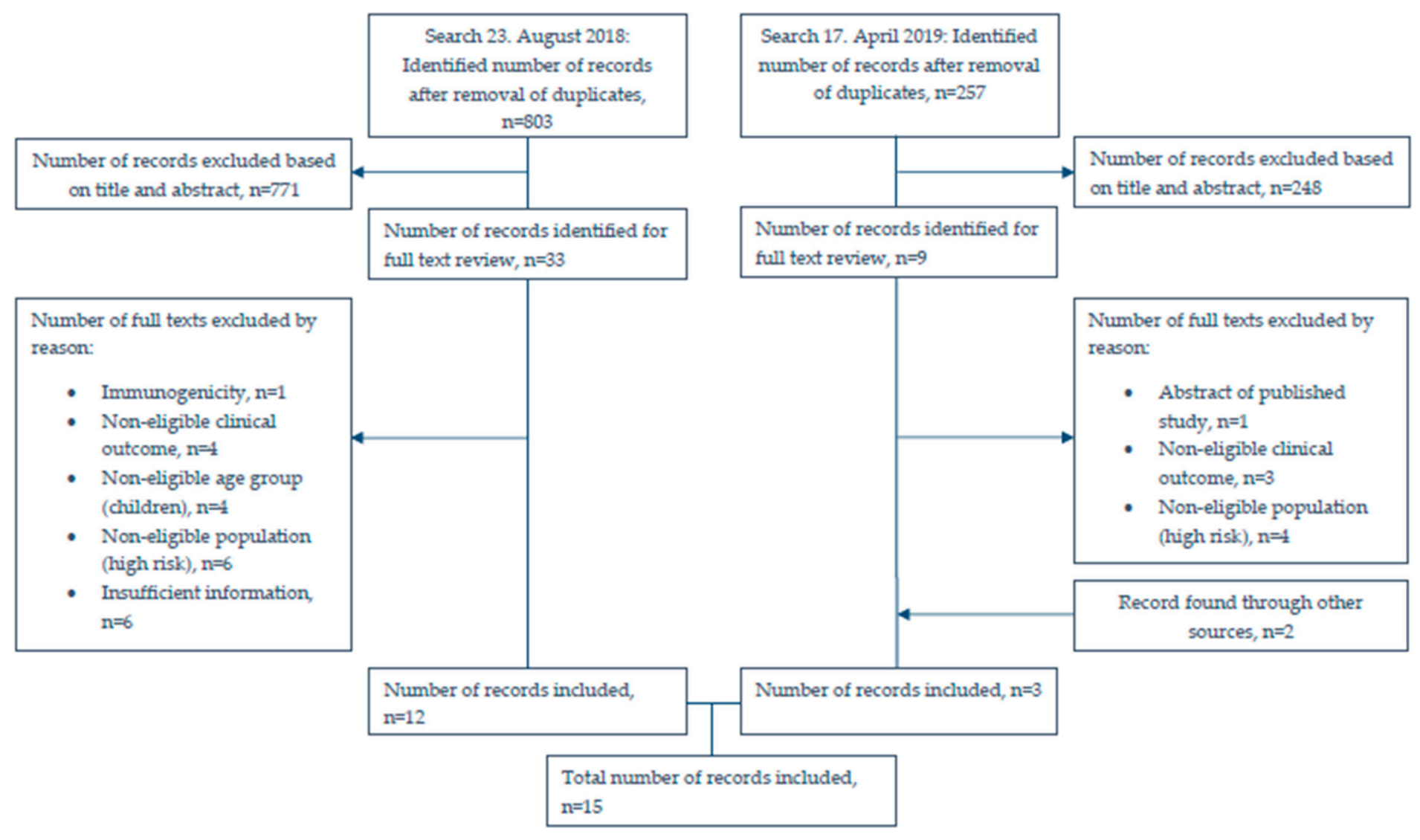

Figure 1. Flowchart of literature search. n: number. 
Table 1. Characteristics of included studies. Y: years. PCV13: 13-valent pneumococcal conjugate vaccine. PPV23: 23-valent pneumococcal polysaccharide vaccine. RCT: randomized controlled trial. TND: Test-negative design. IPD: invasive pneumococcal disease. Pn: Pneumococcal. VT: Vaccine-type.

\begin{tabular}{|c|c|c|c|c|c|c|c|c|}
\hline $\begin{array}{c}\text { Author \& Publication } \\
\text { Year (Ref) }\end{array}$ & Type of Study & Country & Study Period & $\begin{array}{l}\text { Time between Outcome } \\
\text { and Vaccination }\end{array}$ & Outcome & Patient Group & Age (y) & Quality \\
\hline \multicolumn{9}{|c|}{ PCV13 } \\
\hline $\begin{array}{c}\text { Gessner } 2018(11) \\
\text { Huijts } 2017(12) \\
\text { Suaya } 2018(13) \\
\text { Webber } 2017(14) \\
\text { Patterson } 2016(15)\end{array}$ & Post hoc of RCT & Netherlands & 2008-2013 & Up to 5 years & $\begin{array}{l}\text { Pneumonia, pn-pneumonia, } \\
\text { VT-pneumonia, IPD and VT-IPD }\end{array}$ & $\begin{array}{l}\text { Hospitalized } \\
\text { and out-patient }\end{array}$ & $\geq 65$ & NA \\
\hline Vila-Corcoles 2018 (16) & Cohort & Spain & 2015 & Not stated & Pneumonia and pn-pneumonia & Hospitalized & $\geq 50$ & High \\
\hline Kolditz 2018 (17) & Cohort & Germany & 2014-2016 & Up to 5 years & Pneumonia & $\begin{array}{l}\text { Hospitalized } \\
\text { and out-patient }\end{array}$ & $\geq 60$ & High \\
\hline McLaughlin 2018 (18) & TND & US & 2015-2016 & Up to 5 years & VT-pneumonia & Hospitalized & $\geq 65$ & High \\
\hline Prato 2018 (19) & TND & Italy & 2013-2015 & Not stated & $\begin{array}{l}\text { Pn-pneumonia and } \\
\text { VT-pneumonia }\end{array}$ & $\begin{array}{l}\text { Hospitalized } \\
\text { and out-patient }\end{array}$ & $\geq 65$ & Low \\
\hline \multicolumn{9}{|c|}{ PPV23 } \\
\hline Kolditz 2018 (20) & Cohort & Germany & 2010-2011 & Up to 5 years & Pneumonia & $\begin{array}{l}\text { Hospitalized } \\
\text { and out-patient }\end{array}$ & $\geq 60$ & Low \\
\hline Suzuki 2017 (21) & TND & Japan & 2011-2014 & Up to 5 years & $\begin{array}{l}\text { Pn-pneumonia and } \\
\text { VT-pneumonia }\end{array}$ & $\begin{array}{l}\text { Hospitalized } \\
\text { and out-patient }\end{array}$ & $\geq 65$ & High \\
\hline Djennad 2018 (22) & Indirect cohort & UK & $2000-2016$ & Vaccine given at any time & VT-IPD & Hospitalized & $\geq 65$ & High \\
\hline Dominguez 2017 (23) & Case-control & Spain & 2013-2015 & Up to 5 years & Pneumonia & Hospitalized & $\geq 65$ & Low \\
\hline Kondo 2018 (24) & Case-control & Japan & 2009-2014 & Up to 5 years & Pneumonia & Outpatients & $\geq 65$ & Low \\
\hline Kim 2019 (25) & Case-control & South Korea & 2013-2015 & Up to 5 years & $\begin{array}{c}\text { Pn-pneumonia a , VT-pneumonia } \\
\text { a , IPD and VT-IPD }\end{array}$ & Hospitalized & $\geq 65$ & High \\
\hline
\end{tabular}

a non-bacteremic. 


\subsubsection{Polysaccharide Vaccine}

For PPV23 we identified one cohort study from Germany [20], one TND study from Japan [21], one indirect cohort study from United Kingdom (UK) [22] and three case-control studies from Spain [23], Japan [24] and South Korea [25], respectively (Table 1). The German cohort study included almost 739,000 inhabitants aged $\geq 60$ years, retrospectively, using routine insurance data collected between 2010 and 2011. Almost 30\% of these were vaccinated with PPV23 [20]. The Japanese TND study enrolled and analyzed 2036 patients hospitalized with pneumonia between 2001 and 2014. Of the 2036 patients, 419 were categorized as having pneumococcal pneumonia. Sputum analysis identified the majority of these cases [21]. The study from UK included 9847 IPD cases from the enhanced national surveillance of IPD in England and Wales. Among these, 6245 (63\%) had available vaccination information, and were included in VE analysis using the indirect cohort (Broome) method, with 4423 VT IPD cases and 1822 non VT-IPD controls [22]. The case-control study from Spain recruited 1895 CAP cases and an equal amount of controls from 20 different hospitals between 2013 and 2015 [23]. The Japanese case-control study matched 234 outpatients treated for pneumonia between 2009 and 2014 with 438 controls without pneumonia [24]. In South Korea 148 IPD and 557 non-bacteremic pneumococcal pneumonias (NBPP) patients were matched to 295 and 557 hospital controls, respectively. The Korean study identified NBPP cases using sputum culture or urinary antigen test [25].

\subsection{Reported Outcomes}

\subsubsection{Conjugate Vaccine}

Four of the five post hoc studies using CAPITA data provided VE estimates for PCV13 stratified by comorbidities [11-13] and exploratory outcomes [14]. The last post hoc study analyzed the VE of time since vaccination and did not find evidence of waning over the study period [15]. The remaining studies reported VE on the following outcomes: all-cause pneumonia [16,17], pneumococcal pneumonia [16], pneumococcal-CAP [19] and VT-CAP [18,19] (Table 1). None of the observational studies reported on IPD.

For the main outcomes, pneumonia and/or IPD, none of the CAPITA post hoc studies provided any significant new data compared to the original study [2], Hence, we did not quality assess them or extract any VE data on pneumonia or IPD (Table 1).

\subsubsection{Polysaccharide Vaccine}

VE of the PPV23 vaccine was reported on the following outcomes: all-cause pneumonia [20], CAP [23,24], non-bacteremic pneumococcal pneumonia (NBPP) [25], VT-NBPP [25], pneumococcal pneumonia [21] and VT-pneumonia [21], IPD [25] and VT-IPD [22,25] (Table 1).

\subsection{Risk of Bias}

We judged the Italian TND study on PCV13 [19], the German cohort on PPV23 [20], the Spanish case-control on PPV23 [23] and the Japanese case-control study on PPV23 [24] to be of low quality according to NOS criteria (Table $1+$ Table S2). The Italian study had a low inclusion rate of only $10 \%$, stopped before planned time, and did not adjust for confounding factors [19]. The German cohort study did not adequately describe the follow up of the cohort, and the follow up time was only two years [20]. The Spanish case-control used medical records to judge vaccination status. The included cases and controls had a lower vaccination coverage than average in this region and $90 \%$ had one or more comorbidity; this suggests some selection and information bias [23]. In the Japanese case-control study vaccination status and comorbidities were based on self-reporting, introducing a risk of recall bias. Furthermore, there was no information about non-response rate [24].

We judged the remaining observational studies to be of high quality according to the NOS (Table 1 + Table S2). Although we categorized the Spanish cohort study evaluating PCV13 VE as high quality according to the NOS, we found some important problems. The vaccinated cohort was significantly 
sicker and older, with $34 \%$ having an immunocompromising condition and $27 \%$ were 80 years or older. The follow-up period was only one year, and the vaccination rate was only $0.2 \%$ [16].

\subsection{Vaccine efficacy/Effectiveness}

\subsubsection{Conjugate Vaccine}

The Spanish cohort study did not find any protective VE on all-cause pneumonia or pneumococcal pneumonia (Table 2 + Figure 2). The German cohort study found an adjusted VE of 11\% (95\% CI: 3 to 19) against all-cause pneumonia. The American TND study found an adjusted VE of $71 \%$ (95\% CI: 6 to 91 ) against VT-CAP. The Italian TND study found a crude VE of 33\% (95\% CI: -107 to 82$)$ against pneumococcal CAP and a crude VE of 38\% (95\% CI: -132 to 89 ) against VT-CAP.

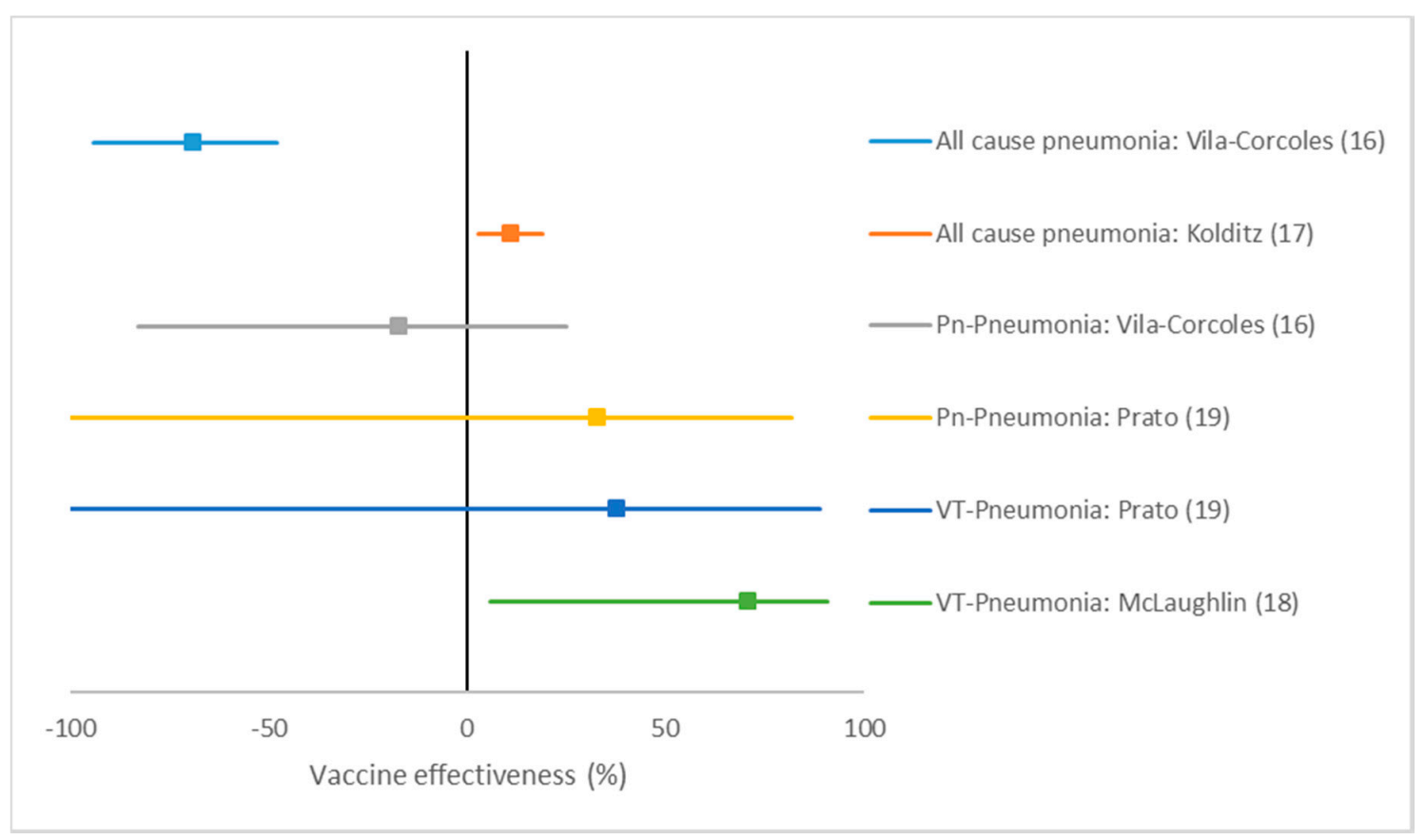

Figure 2. Forest plot of PCV 13 vaccine effectiveness estimates without summary estimate. Pn: pneumococcal. VT: Vaccine-type. 
Table 2. Vaccine effectiveness (VE) percentage with 95\% confidence interval by study, vaccine type and outcome. PCV13: 13-valent pneumococcal conjugate vaccine. PPV23: 23-valent pneumococcal polysaccharide vaccine. PY: Person years. Pn: pneumococcal. VT: vaccine-type. IPD: invasive pneumococcal disease.

\begin{tabular}{|c|c|c|c|c|c|c|c|c|c|}
\hline Author (Ref) & $\begin{array}{l}\text { Episodes or } \\
\text { Cases } \\
\text { Vaccinated }\end{array}$ & $\begin{array}{c}\text { Individuals } \\
\text { Vaccinated or Cases } \\
\text { not Vaccinated }\end{array}$ & $\begin{array}{l}\text { Episodes or } \\
\text { Controls } \\
\text { Vaccinated }\end{array}$ & $\begin{array}{c}\text { Individuals not } \\
\text { Vaccinated } \text { or Controls } \\
\text { not Vaccinated }\end{array}$ & $\begin{array}{c}\text { VE \% } \\
\text { Pneumonia }\end{array}$ & $\begin{array}{c}\text { VE \% } \\
\text { Pn-Pneumonia }\end{array}$ & $\begin{array}{c}\text { VE \% } \\
\text { VT-Pneumonia }\end{array}$ & VE \% IPD & VE \% VT-IPD \\
\hline \multicolumn{10}{|c|}{ PCV 13} \\
\hline \multirow{2}{*}{ Vila-Corcoles (16) } & 228 & 6912 (PY) & 12471 & 1983789 (PY) & $-69(-94$ to -48$)$ & \multirow{3}{*}{-17 (-83 to 25$)$} & & & \\
\hline & 20 & 6912 (PY) & 1628 & 1983789 (PY) & & & & & \\
\hline Kolditz (17) & 532 & 11395 & 1812 & 34185 & 11 (3 to 19 ) & & & & \\
\hline McLaughlin (18) & 3 & 65 & 285 & 1681 & & \multirow{3}{*}{$33(-107$ to 82$)$} & 71 (6 to 91$)$ & & \\
\hline \multirow{2}{*}{ Prato (19) } & 5 & 54 & 15 & 108 & & & & & \\
\hline & 3 & 36 & 17 & 126 & & & $38(-132$ to 89$)$ & & \\
\hline \multicolumn{10}{|c|}{ PPV 23} \\
\hline Kolditz (20) & 7501 & 213431 & 23243 & 640293 & $3(1$ to 6$)$ & \multirow{4}{*}{27 (3 to 46$)$} & & & \multirow{9}{*}{27 (17 to 35$)$} \\
\hline \multirow{2}{*}{ Suzuki (21) } & 95 & 214 & 427 & 745 & & & & & \\
\hline & 58 & 146 & 427 & 745 & & & 34 (6 to 53$)$ & & \\
\hline Djennad (22) & 2741 & 1682 & 1288 & 534 & & & & & \\
\hline Dominguez (23) & 259 & 1636 & 272 & 1623 & 15 ( -3 to 30$)$ & & & & \\
\hline Kondo (24) & 64 & 170 & 131 & 307 & $16(-30$ to 46$)$ & \multirow{5}{*}{$10(-15$ to 30$)$} & & & \\
\hline \multirow{4}{*}{ Kim (25) } & 231 & 326 & 247 & 310 & & & & & \\
\hline & 106 & 137 & 247 & 310 & & & $2(-39$ to 26$)$ & \multirow{3}{*}{29 ( -6 to 52$)$} & \\
\hline & 54 & 94 & 130 & 165 & & & & & \\
\hline & 21 & 43 & 130 & 165 & & & & & $42(-2$ to 67$)$ \\
\hline
\end{tabular}




\subsubsection{Polysaccharide Vaccine}

The German cohort study did not present any relative measure in their results section, but using their numbers we calculated an adjusted (propensity score matched) VE of 3\% (95\% CI: 1 to 6) against all-cause pneumonia (Table 2 + Figure 3). The Japanese TND study found an adjusted VE of $27 \%(95 \%$ CI: 3 to 46 ) against pneumococcal pneumonia and a VE of $34 \%$ (95\% CI: 6 to 53) against VT-pneumonia. The Spanish case-control study found an adjusted VE of 15\% (95\% CI: -3 to 30) against CAP. The Japanese case-control study found a similar adjusted VE of $16 \%(95 \% \mathrm{CI}$ : -30 to 46$)$ against CAP. The South Korean case-control study found an adjusted VE of 10\% (95\% CI: -15 to 30) against NBPP and $29 \%$ (95\% CI: -6 to 52$)$ against IPD. For VT-NBPP and VT-IPD the VE was $-2 \%$ (95\% CI: -40 to 26) and $42 \%$ (95\% CI: -2 to 67 ), respectively. The UK study reported a VE of $27 \%$ (95\% CI: 17 to 35 ) against VT-IPD.

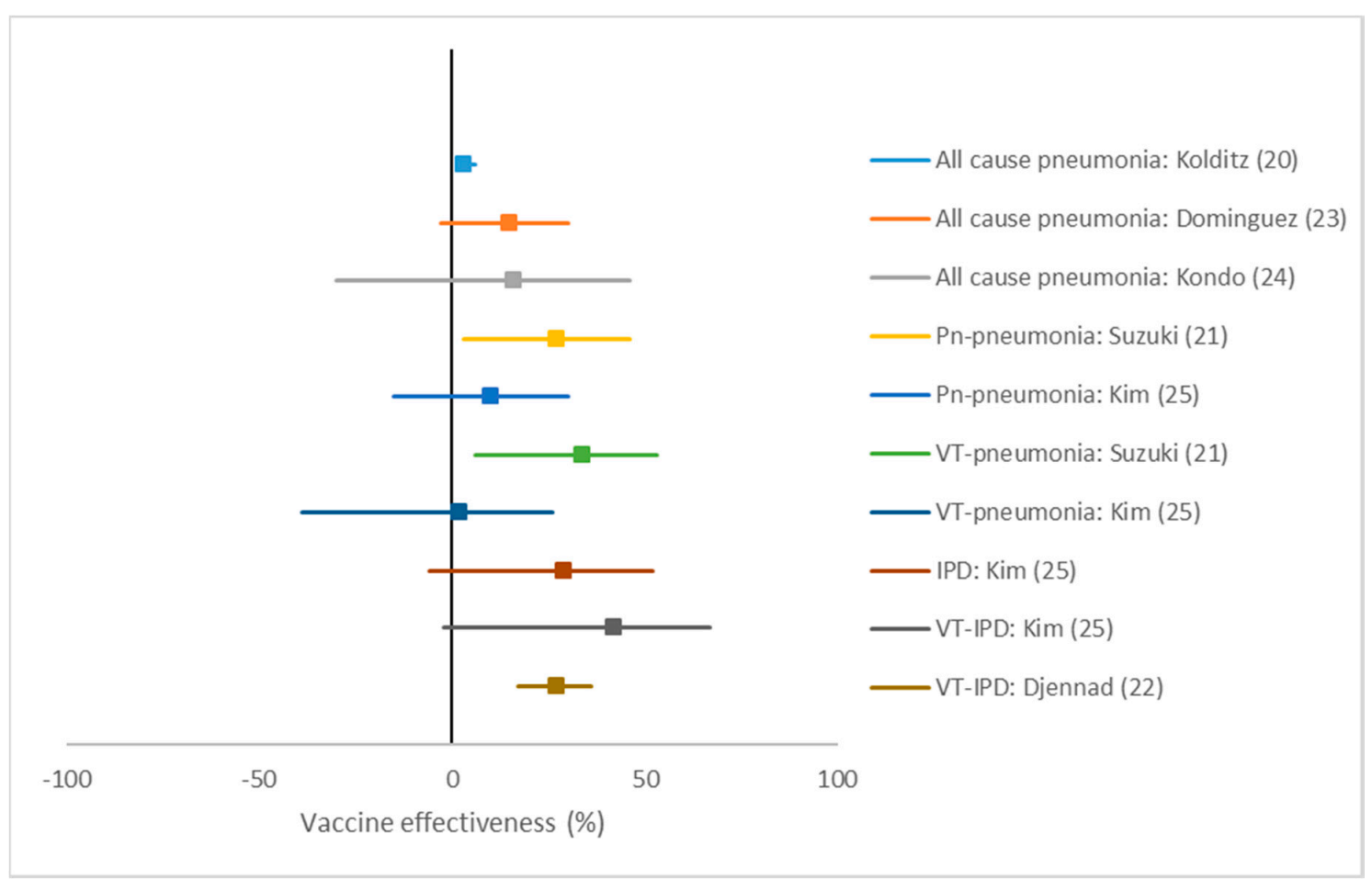

Figure 3. Forest plot of PPV23 vaccine effectiveness estimates without summary estimate. Pn: pneumococcal. VT: Vaccine-type. IPD: invasive pneumococcal disease.

3.5. Age

Five studies on PPV23 [20-23,25] reported VE stratified by age groups (Table 3). For PPV23 there seems to be a decreasing VE with increasing age, and only one study [22] reported a significant VE in the oldest age group ( $\geq 85$ years). For PCV13, one study showed decreasing VE by age results [16], while another did not find any difference [17] (Table 3). 
Table 3. Vaccine effectiveness (VE) percentage with 95\% confidence interval by vaccine and age group. VE: Vaccine effectiveness. PPV23: 23 -valent polysaccharide vaccine. PCV13: 13-valent conjugate vaccine. Pn: pneumococcal. VT: vaccine-type. IPD: invasive pneumococcal disease.

\begin{tabular}{|c|c|c|c|c|c|c|c|}
\hline Author (Ref) & Outcome & Number of Cases $\S$ & All & $50-59$ & $70-74$ & $75-79$ & $\geq 85$ \\
\hline \multicolumn{8}{|c|}{ PCV 13} \\
\hline \multirow{2}{*}{$\begin{array}{c}\text { Vila-Corcoles } \\
\text { (16) }\end{array}$} & Pneumonia & 12699 & $-69(-94$ to -48$)$ & $-21(-72$ to 15$)$ & \multirow{2}{*}{\multicolumn{3}{|c|}{$\begin{array}{l}-76(-104 \text { to }-52){ }^{\mathrm{A}} \\
-32(-118 \text { to } 19)\end{array}$}} \\
\hline & Pn-Pneumonia & 1648 & $-17(-83$ to 25$)$ & $42(-67$ to 80$)$ & \multirow{2}{*}{$8(-6$ to 19$)$} & & \multirow[b]{2}{*}{$10(-3 \text { to } 28)^{\mathrm{B}}$} \\
\hline Kolditz (17) & Pneumonia & 2344 & $11(3$ to 19$)$ & - & & & \\
\hline \multicolumn{8}{|c|}{ PPV 23} \\
\hline Kolditz (20) & Pneumonia & 30744 & $3(1$ to 6$)$ & - & 2 ( -6 to 2$)$ & \multicolumn{2}{|c|}{$0(-4 \text { to } 3)^{C}$} \\
\hline \multirow{2}{*}{ Suzuki (21) } & Pn-Pneumonia & 419 & 27 (3 to 46$)$ & - & $32(-21$ to 62$)$ & \multicolumn{2}{|c|}{$24(-6 \text { to } 46)^{\mathrm{D}}$} \\
\hline & VT-Pneumonia & 272 & $34(6$ to 53$)$ & - & $40(-6$ to 69$)$ & \multicolumn{2}{|c|}{$28(-10 \text { to } 53)^{\mathrm{D}}$} \\
\hline Djennad (22) & VT-IPD & 4423 & 27 (17 to 35$)$ & - & $31(16 \text { to } 44)^{\mathrm{E}}$ & $17(-3 \text { to } 32)^{\mathrm{F}}$ & $34(17 \text { to } 47)^{\mathrm{G}}$ \\
\hline Dominguez (23) & Pneumonia & 1895 & $15(-3$ to 30$)$ & - & $24(-3 \text { to } 43)^{\mathrm{H}}$ & $12(-22 \text { to } 36)^{\mathrm{I}}$ & $0(-58$ to 37$) \mathrm{J}$ \\
\hline \multirow[t]{4}{*}{ Kim (25) } & Pn-Pneumonia & 557 & $10(-15$ to 30$)$ & - & 35 (2 to 57$)$ & \multicolumn{2}{|c|}{$-13(-56 \text { to } 18)^{\mathrm{K}}$} \\
\hline & VT-Pneumonia & 243 & $-2(-40$ to 26$)$ & - & $21(-31$ to 52$)$ & \multicolumn{2}{|c|}{$-35(-107$ to 12$)$} \\
\hline & IPD & 148 & $29(-6$ to 52$)$ & - & $57(19$ to 78$)$ & \multicolumn{2}{|c|}{$7(-74$ to 50$)$} \\
\hline & VT-IPD & 64 & $42(-2$ to 67$)$ & - & $70(25$ to 88$)$ & \multicolumn{2}{|c|}{$-20(-184$ to 60$)$} \\
\hline
\end{tabular}

${ }^{\S}$ Number of cases with outcome. ${ }^{\mathrm{A}} 50 \%$ (of total included participants). ${ }^{\mathrm{B}} 41 \% .{ }^{\mathrm{C}} 35 \%$. ${ }^{\mathrm{D}} 78 \%$. ${ }^{\mathrm{E}} 38 \% .{ }^{\mathrm{F}} 37 \% .{ }^{\mathrm{G}} 25 \% .{ }^{\mathrm{H}} 31 \%$. ${ }^{\mathrm{I}} 47 \%$. ${ }^{\mathrm{J}} 22 \%$. ${ }^{\mathrm{K}} 58 \%$. 


\section{Discussion}

\subsection{General Comments}

Studies evaluating the VE of pneumococcal vaccines for the prevention of pneumonia are difficult to compare due to a lack of a standardized outcome definitions and diagnostic tools. The outcomes in the included studies in our systematic search ranged from all-cause pneumonia to VT-IPD. The cohort studies used routine data based on International Classification of Disease codes, while all other studies validated their cases using comparable clinical criteria (i.e., radiological findings combined with clinical symptoms and paraclinical observations). Most studies combined bacteremic and non-bacteremic pneumonias, and only one study analyzed non-bacteremic pneumonias.

The usual way to identify pneumococcal bacteremia is by blood culture; however, this is not a feasible way to identify non-bacteremic pneumococcal pneumonias [26]. The studies in our review used several different tests to identify pneumococcal pneumonias; blood culture, sputum culture, sputum PCR, and urinary antigen tests, either as the BinaxNOW, a non serotype-specific test, or as the serotype-specific SSUAD developed to identify PCV13 serotypes. Some studies used a wide range of tests, while others used one or two.

Other factors that can explain the large differences in VE are serotype distribution, background pneumococcal vaccination coverage, different study designs, characteristics of included participants, time since vaccination, and follow-up time.

The main limitation of this review is the short time span of the systematic search. The low number of new publications did not permit meta-analyses stratified by vaccine, study design and outcome. However, our results can be used in conjunction with earlier studies not included in this review [2-7] (Table S1). Another limitation is the inconsistent use of age groups across studies, making it difficult to compare VE by age.

\subsection{Evidence Base for PCV13}

The VE in observational studies on PCV13 ranges from a negative VE (adjusted VE -69\%) against all-cause pneumonia to a high protective VE of $71 \%$ against VT-CAP. All of these studies were conducted in the period 2014 to 2016, and in regions where PCV13 is a part of the routine childhood immunization schedule.

The post hoc studies using CAPITA data provided some interesting information. Huijts et al. retrospectively obtained information on comorbidities from medical records (from GPs, hospitals) for participants who were identified with vaccine-type CAP $(n=139)$, and from ICPC codes from general practitioners for 40427 CAPITA participants. The number of individuals with comorbidities was higher when data were obtained from medical records rather than from self-report [12]. This could suggest that the population in CAPITA actually was less healthy than previously reported. Patterson et al. studied the VE of time since vaccination, and did not find any evidence of waning over the study period of four years [15].

The TND study from the US had a surprisingly high VE against VT-CAP [18]. However, the wide confidence intervals (reflecting a relatively low amount of VT-CAP cases) does overlap with results from CAPITA. Eight percent of the VT-CAP cases were bacteremic, which is a higher proportion than in the other two TND-studies (where the proportion was one percent). When restricted to non-bacteremic VT-CAP, the VE was a bit lower and became statistically non-significant $68 \%$ ( $95 \%$ CI: -6 to 90 ). The pneumococcal vaccines have consistently been found to provide better protection against invasive than non-invasive disease, and if the proportion of bacteremic pneumonia is high compared to non-bacteremic pneumonia one would expect a higher VE. Furthermore, the median time since vaccination was only 157 days. This short time since vaccination might also contribute to the high observed VE.

The Italian TND study mixed different diagnostic tools, included only $10 \%$ of eligible patients, stopped before planned time, and did not adjust for underlying factors [19]. This has probably 
introduced detection and selection bias. However, the calculated VE is similar to the VE found in CAPITA and overlaps with the VE from the well-conducted TND from the US. The TND design also has several methodological advantages as it can reduce health care seeking bias and misclassification bias [27]. The VE against pneumococcal pneumonia is relatively high (33\%) and almost the same as against VT pneumonia (38\%). In the study, PCV13 serotypes caused $66 \%$ of the pneumococcal pneumonias. Although this might seem unlikely in a region with PCV13 childhood vaccination, an IPD surveillance study from Italy between 2008 and 2014 only showed a small, and not statistically significant, reduction in PCV13 serotypes for those aged $<64$ years [28].

In the large Spanish cohort study, only $0.2 \%$ were PCV vaccinated [16]. The authors found no protective VE of the vaccine. Actually, they found an increased risk of pneumonia after adjustment in the vaccinated group. However, the vaccinated group was significantly older, with more comorbidities, and a higher uptake of the polysaccharide and influenza vaccine, and this could have introduced selection bias. The PPV23 coverage amongst elderly in this region of Spain have been high for many years. This could also lead to a lower estimated VE of the conjugate vaccine in Spain compared to CAPITA results from the Netherlands with a very low background PPV23 vaccination rate in the elderly population. Finally, this region of Spain has had a large reduction in circulating PCV13 serotypes due to the indirect effect of the PCV childhood immunization [29]. In a population with a significant reduction in PCV13 serotypes, the preventative potential of PCV13 is lower than in a population with a high proportion of circulating PCV13 serotypes.

\subsection{Evidence Base for PPV23}

The VE of the PPV23 vaccine in our review ranged from 3\% to $16 \%$ against all-cause pneumonia (Table 2). This overlaps with results from a systematic review by Kraicer-Melamed et al., where the authors found a small but non-significant protective VE of $17 \%$ in cohort studies and $7 \%$ in case-control studies [5]. Similarly, Tin Tin Htar et al. found a non-significant VE for any-CAP hospitalization of $10 \%$ in observational studies [7].

The VE against pneumococcal pneumonia in our review (Table 2) also overlaps with results from some previous systematic reviews. Falkenhorst reported a VE of $48 \%$ in cohort studies, $53 \%$ in a case-control study and 37 in a TND study [4], Kraicer-Melamed et al. reported a range between 5\% and $45 \%$ for cohort studies and $48 \%$ in one case-control study [5], while Tin Tin Htar reported a VE of $45-53 \%$ in observational studies [7].

No other systematic reviews have reported on the VE on VT-pneumonia. In our review we found two recent studies on this topic, a TND study from Japan [21], and a case-control study from South Korea [25]. Both studies evaluated VE of PPV23 in an elderly Asiatic population.

The TND study from Japan found a VE of 34\% against VT-pneumonia [21]. This study used a range of different diagnostic tools. Combining different tests can increase the overall sensitivity but decrease the overall specificity. A modeling study suggests that a decreased specificity might underestimate the true VE in observational studies [30]. Thus, one could consider the estimated VE in the Japanese study a minimum number. Sputum samples identified 208 of the 419 pneumococcal positive laboratory samples. Some of these patients might just be carriers, and this might overestimate the number of positive pneumococcal infections. In that respect, it is noteworthy that sensitivity analyses with different cut-off values for the DNA load found similar results even with a 100-fold increase. The authors found reduced VE of PPV23 by time since vaccination, with only the first two years since vaccination having a statistically significant VE (Table S3). The UK IPD surveillance study also reported a lower VE point estimate for PPV23 as the time since vaccination increased [22]. However, although the point estimate decreased from $41 \%$ for those vaccinated within two years to $23 \%$ for those vaccinated more than five years ago, the VE against VT-IPD remained statistically significant also after five years (Table S3).

The South Korean case-control study did not find significant VE against NBPP or IPD for all age groups 65 years or older [25]. However, when stratifying by age, the authors found significant 
protection in patients aged 65-74 years against both outcomes (Table 3), indicating an age dependent PPV23 VE. Fifty-eight percent of the total included population was 75 years or older, and this could have affected the overall results for all patients aged 65 or older. This age dependent VE of the PPV23 was also noted in the Japanese TND study [21] and Spanish case-control study [23] (Table 3). Similarly, CAPITA did not find a significantly protective VE of PCV13 against the first episode of VT CAP in the study population aged 75 years or older (VE 32\% (95\% CI: -22 to 63)) [2]. The South Korean study was the only study that restricted their pneumonia cases to non-bacteremic cases.

The VE on IPD in the studies included in our review also overlaps with some previous reviews on observational studies. Both Falkenhorst [4] and Kraicer-Melamed et al. [5] reported an estimated VE of around $50 \%$ with a $95 \%$ CI range of 15 to 74 .

\subsection{Future Perspectives}

In our opinion, future studies on VE of pneumococcal vaccines should include serotype-specific information. In combination with local serotype distribution data on disease, this information can help medical and public health professionals tailor vaccination strategies against pneumococcal disease. However, in order to achieve this VE data, readily available serotype-specific diagnostic tools for non-bacteremic disease are needed. Although the SSUAD developed by Pfizer is an example of this, it can only identify serotypes included in PCV13, and it is only available for research purposes.

\section{Conclusions}

There are strengths and weaknesses of all the included studies. We chose to include a wide range of outcomes and study designs as all of these outcomes are interesting from a public health perspective, and may add evidence in combination with earlier studies not included in this review. New high-quality observational studies indicate protective VE for both the PPV23 [21] and the PCV13 [18] against VT pneumonia. The results from those studies have overlapping CI with the results from CAPITA [2]. Our estimates for the protective VE of PPV23 on pneumonia and pneumococcal pneumonia overlap with results from previously published reviews [3-7]. Some of the results indicate that the VE on the PPV23 is best in younger age groups, and that it decreases over time.

Supplementary Materials: The following are available online at http://www.mdpi.com/2076-0817/9/4/259/s1, Table S1: Overview of recent meta analyses of PPV23 efficacy/effectiveness. Table S2: Quality of included studies according to Newcastle-Ottawa scale and threshold categorized from Agency for Healthcare Research and Quality (AHRQ). Table S3: Vaccine efficacy/effectiveness (VE) percentage including 95\% confidence interval of the 23-valent polysaccharide vaccine by time since vaccination. Pn: pneumococcal. VT: vaccine-type. IPD: invasive pneumococcal disease. Appendix A: Search strategy. Appendix B: data extraction form. Appendix C: PICO.

Author Contributions: Conceptualization, J.D.B., B.A.W. and J.S.; methodology, J.D.B., B.A.W., D.F.V., H.-C.S., P.V.-B., A.R., J.S.; writing — original draft preparation, J.D.B.; writing—review and editing, J.D.B., B.W., D.F.V., H.-C.S., P.V.-B., A.R., J.S. All authors have read and agreed to the published version of the manuscript.

Funding: This research received no external funding.

Conflicts of Interest: Hans-Christian Slotved is involved with projects supported by Pfizer.

\section{Abbreviations}

CAP: Community acquired pneumonia. IPD: invasive pneumococcal disease. NBPP: non-bacteremic pneumococcal pneumonia. NOS: Newcastle-Ottawa Scale. PCV13: 13-valent conjugate vaccine. Pn: pneumococcal. PPV23: 23-valent polysaccharide vaccine. RCT: randomized controlled trials. TND: test-negative design. VE: Vaccine efficacy (clinical trials) or vaccine effectiveness (observational studies). VT: vaccine-type.

\section{References}

1. Wroe, P.C.; Finkelstein, J.A.; Ray, G.T.; Linder, J.A.; Johnson, K.M.; Rifas-Shiman, S.; Moore, M.R.; Huang, S.S. Aging population and future burden of pneumococcal pneumonia in the United States. J. Infect. Dis. 2012, 205, 1589-1592. [CrossRef] [PubMed] 
2. Bonten, M.J.; Huijts, S.M.; Bolkenbaas, M.; Webber, C.; Patterson, S.; Gault, S.; van Werkhoven, C.H.; van Deursen, A.M.; Sanders, E.A.; Verheij, T.J.; et al. Polysaccharide conjugate vaccine against pneumococcal pneumonia in adults. N. Engl. J. Med. 2015, 372, 1114-1125. [CrossRef] [PubMed]

3. Diao, W.Q.; Shen, N.; Yu, P.X.; Liu, B.B.; He, B. Efficacy of 23-valent pneumococcal polysaccharide vaccine in preventing community-acquired pneumonia among immunocompetent adults: A systematic review and meta-analysis of randomized trials. Vaccine 2016, 34, 1496-1503. [CrossRef] [PubMed]

4. Falkenhorst, G.; Remschmidt, C.; Harder, T.; Hummers-Pradier, E.; Wichmann, O.; Bogdan, C. Effectiveness of the 23-Valent Pneumococcal Polysaccharide Vaccine (PPV23) against Pneumococcal Disease in the Elderly: Systematic Review and Meta-Analysis. PLOS ONE 2017, 12, e0169368. [CrossRef] [PubMed]

5. Kraicer-Melamed, H.; O'Donnell, S.; Quach, C. The effectiveness of pneumococcal polysaccharide vaccine 23 (PPV23) in the general population of 50 years of age and older: A systematic review and meta-analysis. Vaccine 2016, 34, 1540-1550, Corrigendum in 2016, 34, 4083-4084, doi:10.1016/j.vaccine.2016.06.045. [CrossRef] [PubMed]

6. Schiffner-Rohe, J.; Witt, A.; Hemmerling, J.; von Eiff, C.; Leverkus, F.W. Efficacy of PPV23 in Preventing Pneumococcal Pneumonia in Adults at Increased Risk-A Systematic Review and Meta-Analysis. PLoS ONE 2016, 11, e0146338. [CrossRef] [PubMed]

7. Tin Tin Htar, M.; Stuurman, A.L.; Ferreira, G.; Alicino, C.; Bollaerts, K.; Paganino, C.; Reinert, R.R.; Schmitt, H.J.; Trucchi, C.; Vestraeten, T.; et al. Effectiveness of pneumococcal vaccines in preventing pneumonia in adults, a systematic review and meta-analyses of observational studies. PLoS ONE 2017, 12, e0177985. [CrossRef]

8. Higgins, J.P.T.; Altman, D.G.; Gøtzsche, P.C.; Jüni, P.; Moher, D.; Oxman, A.D.; Savović, J.; Schulz, K.F.; Weeks, L.; Sterne, J.A.C. The Cochrane Collaboration's tool for assessing risk of bias in randomised trials. BMJ 2011, 343, d5928. [CrossRef]

9. Wells, G.A.; Shea, B.; Higgins, J.P.; Sterne, J.; Tugwell, P.; Reeves, B.C. Checklists of methodological issues for review authors to consider when including non-randomized studies in systematic reviews. Res. Synth. Methods 2013, 4, 63-77. [CrossRef]

10. Wells, G.A.; Shea, B.; O'Connell, D.; Peterson, J.; Welch, V.; Losos, M.; Tugwell, P. The Newcastle-Ottawa Scale (NOS) for Assessing the Quality of Nonrandomised Studies in Meta-Analyses. Available online: http://www.ohri.ca/programs/clinical_epidemiology/oxford.asp (accessed on 11 November 2019).

11. Gessner, B.D.; Jiang, Q.; Van Werkhoven, C.H.; Sings, H.L.; Webber, C.; Scott, D.; Neuzil, K.M.; O’Brien, K.L.; Wunderink, R.G.; Grobbee, D.E.; et al. A public health evaluation of 13-valent pneumococcal conjugate vaccine impact on adult disease outcomes from a randomized clinical trial in the Netherlands. Vaccine 2018. [CrossRef]

12. Huijts, S.M.; van Werkhoven, C.H.; Bolkenbaas, M.; Grobbee, D.E.; Bonten, M.J. Post-hoc analysis of a randomized controlled trial: Diabetes mellitus modifies the efficacy of the 13-valent pneumococcal conjugate vaccine in elderly. Vaccine 2017, 35, 4444-4449. [CrossRef] [PubMed]

13. Suaya, J.A.; Jiang, Q.; Scott, D.A.; Gruber, W.C.; Webber, C.; Schmoele-Thoma, B.; Hall-Murray, C.K.; Jodar, L.; Isturiz, R.E. Post hoc analysis of the efficacy of the 13-valent pneumococcal conjugate vaccine against vaccine-type community-acquired pneumonia in at-risk older adults. Vaccine 2018, 36, 1477-1483. [CrossRef] [PubMed]

14. Webber, C.; Patton, M.; Patterson, S.; Schmoele-Thoma, B.; Huijts, S.M.; Bonten, M.J.; CAPiTA Study Group. Exploratory efficacy endpoints in the Community-Acquired Pneumonia Immunization Trial in Adults (CAPiTA). Vaccine 2017, 35, 1266-1272. [CrossRef] [PubMed]

15. Patterson, S.; Webber, C.; Patton, M.; Drews, W.; Huijts, S.M.; Bolkenbaas, M.; Gruber, W.C.; Scott, D.A.; Bonten, M.J.M. A post hoc assessment of duration of protection in CAPiTA (Community Acquired Pneumonia immunization Trial in Adults). Trials Vaccinol. 2016, 5, 92-96. [CrossRef]

16. Vila-Corcoles, A.; Ochoa-Gondar, O.; de Diego, C.; Satue, E.; Aragón, M.; Vila-Rovira, A.; Gomez-Bertomeu, F.; Magarolas, R.; Figuerola-Massana, E.; Raga, X.; et al. Evaluating clinical effectiveness of 13-valent pneumococcal conjugate vaccination against pneumonia among middle-aged and older adults in Catalonia: Results from the EPIVAC cohort study. BMC Infect. Dis. 2018, 18, 196. [CrossRef] [PubMed]

17. Kolditz, M.; Schmitt, J.; Pletz, M.W.; Tesch, F. Impact of the 13-valent pneumococcal conjugate vaccine on incidence of all-cause pneumonia in adults aged $>=60$ years-a population-based retrospective cohort study. Clin. Infect. Dis. 2018, 21, 21. [CrossRef] 
18. McLaughlin, J.M.; Jiang, Q.; Isturiz, R.E.; Sings, H.L.; Swerdlow, D.L.; Gessner, B.D.; Carrico, R.M.; Peyrani, P.; Wiemken, T.L.; Mattingly, W.A.; et al. Effectiveness of 13-Valent Pneumococcal Conjugate Vaccine Against Hospitalization for Community-Acquired Pneumonia in Older US Adults: A Test-Negative Design. Clin. Infect. Dis. 2018. [CrossRef]

19. Prato, R.; Fortunato, F.; Cappelli, M.G.; Chironna, M.; Martinelli, D. Effectiveness of the 13-valent pneumococcal conjugate vaccine against adult pneumonia in Italy: A case-control study in a 2-year prospective cohort. BMJ Open 2018, 8, e019034. [CrossRef]

20. Kolditz, M.; Schmitt, J.; Pletz, M.W.; Tesch, F. Impact of pneumococcal polysaccharide vaccine on incidence and mortality after pneumonia in adults aged $>/=60$ years-a population-based retrospective cohort study. Clin. Microbiol. Infect. 2018, 24, 500-504. [CrossRef]

21. Suzuki, M.; Dhoubhadel, B.G.; Ishifuji, T.; Yasunami, M.; Yaegashi, M.; Asoh, N.; Ishida, M.; Hamaguchi, S.; Aoshima, M.; Ariyoshi, K.; et al. Adult Pneumonia Study, Group-Japan. Serotype-specific effectiveness of 23-valent pneumococcal polysaccharide vaccine against pneumococcal pneumonia in adults aged 65 years or older: A multicentre, prospective, test-negative design study. Lancet Infect. Dis. 2017, 17, 313-321. [CrossRef]

22. Djennad, A.; Ramsay, M.E.; Pebody, R.; Fry, N.K.; Sheppard, C.; Ladhani, S.N.; Andrews, N.J. Effectiveness of 23-Valent Polysaccharide Pneumococcal Vaccine and Changes in Invasive Pneumococcal Disease Incidence from 2000 to 2017 in Those Aged 65 and Over in England and Wales. EClinicalMedicine 2018, 6, 42-50. [CrossRef] [PubMed]

23. Domínguez, À.; Soldevila, N.; Toledo, D.; Torner, N.; Force, L.; Pérez, M.J.; Martin, V.; Rodriguez-Rojas, L.; Astray, J.; Egurrola, M.; et al. Effectiveness of 23-valent pneumococcal polysaccharide vaccination in preventing community-acquired pneumonia hospitalization and severe outcomes in the elderly in Spain. PLoS ONE 2017, 12, e0171943. [CrossRef] [PubMed]

24. Kondo, K.; Suzuki, K.; Washio, M.; Ohfuji, S.; Fukushima, W.; Maeda, A.; Hirota, Y. Effectiveness of 23-valent pneumococcal polysaccharide vaccine and seasonal influenza vaccine for pneumonia among the elderly-Selection of controls in a case-control study. Vaccine 2017, 35, 4806-4810. [CrossRef] [PubMed]

25. Kim, J.H.; Chun, B.C.; Song, J.Y.; Kim, H.Y.; Bae, I.-G.; Kim, D.-M.; Choi, Y.H.; Jun, Y.H.; Choi, W.S.; Kang, S.H.; et al. Direct effectiveness of pneumococcal polysaccharide vaccine against invasive pneumococcal disease and non-bacteremic pneumococcal pneumonia in elderly population in the era of pneumococcal conjugate vaccine: A case-control study. Vaccine 2019, 37, 2797-2804. [CrossRef] [PubMed]

26. Said, M.A.; Johnson, H.L.; Nonyane, B.A.; Deloria-Knoll, M.; O’Brien, K.L.; Andreo, F.; Beovic, B.; Blanco, S.; Boersma, W.G.; Boulware, D.R.; et al. Estimating the burden of pneumococcal pneumonia among adults: A systematic review and meta-analysis of diagnostic techniques. PLoS ONE 2013, 8, e60273. [CrossRef] [PubMed]

27. Fukushima, W.; Hirota, Y. Basic principles of test-negative design in evaluating influenza vaccine effectiveness. Vaccine 2017, 35, 4796-4800. [CrossRef]

28. D'Ancona, F.; Caporali, M.G.; Del Manso, M.; Giambi, C.; Camilli, R.; D’Ambrosio, F.; Del Grosso, M.; Iannazzo, S.; Rizzuto, E.; Pantosti, A. Invasive pneumococcal disease in children and adults in seven Italian regions after the introduction of the conjugate vaccine, 2008-2014. Epidemiol. Prev. 2015, 39, 134-138.

29. Hanquet, G.; Krizova, P.; Valentiner-Branth, P.; Ladhani, S.N.; Nuorti, J.P.; Lepoutre, A.; Mereckiene, J.; Knol, M.; Winje, B.A.; Ciruela, P.; et al. Effect of childhood pneumococcal conjugate vaccination on invasive disease in older adults of 10 European countries: Implications for adult vaccination. Thorax 2019, 74, 473-482. [CrossRef]

30. Orenstein, E.W.; De Serres, G.; Haber, M.J.; Shay, D.K.; Bridges, C.B.; Gargiullo, P.; Orenstein, W.A. Methodologic issues regarding the use of three observational study designs to assess influenza vaccine effectiveness. Int. J. Epidemiol. 2007, 36, 623-631. [CrossRef]

(C) 2020 by the authors. Licensee MDPI, Basel, Switzerland. This article is an open access article distributed under the terms and conditions of the Creative Commons Attribution (CC BY) license (http://creativecommons.org/licenses/by/4.0/). 